\title{
Does phonological encoding in speech production always follow the retrieval of semantic knowledge? Electrophysiological evidence for parallel processing
}

\author{
Rasha Abdel Rahman*, Werner Sommer \\ Humboldt University, Berlin, Germany \\ Accepted 23 December 2002
}

\begin{abstract}
In this article a new approach to the distinction between serial/contingent and parallel/independent processing in the human cognitive system is applied to semantic knowledge retrieval and phonological encoding of the word form in picture naming. In two-choice go/nogo tasks pictures of objects were manually classified on the basis of semantic and phonological information. An additional manipulation of the duration of the faster and presumably mediating process (semantic retrieval) allowed to derive differential predictions from the two alternative models. These predictions were tested with two event-related brain potentials (ERPs), the lateralized readiness potential (LRP) and the N200. The findings indicate that phonological encoding can proceed in parallel to the retrieval of semantic features. A suggestion is made how to accommodate these findings with models of speech production.

(C) 2003 Elsevier Science B.V. All rights reserved.
\end{abstract}

Theme: Neural basis of behavior

Topic: Cognition: language

Keywords: Speech production; Serial versus parallel processing; Semantics and phonology; LRP

\section{Introduction}

Speaking can be conceived of as the translation of an abstract, conceptual representation into a phonological word form that can be articulated. It is widely accepted that this process involves at least three components of conceptual-semantic, syntactic, and phonological encoding $[10,13,18,19]$. For example, in object naming-which is assumed to be a representative instance of speech production - the lexical entry (lemma) is selected on the basis of prior conceptual processing. After lemma selection, the respective word form becomes available. Although most models of speech production agree that object naming is conceptually mediated, the extent to which lemma selec-

\footnotetext{
*Corresponding author. Max Planck Institute for Psycholinguistics, PO Box 310, 6500 AH Nijmegen, The Netherlands. Tel.: +31-24-3521-320; fax: +31-24-3521-213.

E-mail address: rasha.abdel-rahman@mpi.nl (R. Abdel Rahman).
}

tion and hence phonological encoding is contingent on the retrieval of semantic information is unclear. One class of models suggests that lexical access is mediated by a combination of basic semantic concept nodes which represent the meaning of words $[3,11]$. In picture naming, for example, the word $\operatorname{dog}$ is retrieved on the basis of primitive semantic features such as ANIMATE, HERBIVOROUS, FELINE. In contrast to this serial account it has been proposed that retrieval of an abstract concept node is sufficient for object naming. Semantic features such as ANIMATE, HERBIVOROUS and FELINE are not necessary for accessing the word $d o g$; any semantic feature can therefore be retrieved in parallel to the word form [19]. The use of the term 'parallel' for referring to this theoretical position might be somewhat confusing because the model of Levelt and colleagues [19] is typically characterized as 'serial discrete'. However, the terms serial and parallel bear on different aspects of the model. It is parallel with respect to the retrieval of 
semantic features on the one hand and lemma selection and phonological encoding on the other hand, both processes being mediated by an abstract concept node. It is, however, serial discrete with respect to the transmission of information from a single lemma candidate to the word's phonological form. The aim of the present study was to investigate whether phonological encoding in object naming does in fact depend on prior retrieval of basic semantic knowledge (serial account) or whether retrieval of both types of information can be initiated at about the same time (parallel account).

Recent evidence on the time course of semantic, syntactic and phonological processes during speech production has come from a series of studies by Van Turennout, et al. [31,32] and Schmitt et al. [27,28]. In these studies eventrelated brain potentials (ERPs) were recorded while participants performed a so-called 2-choice go/nogo task. Pictures of objects were classified, for example, as living (e.g., a tiger) or non-living (e.g., a chair) by means of key presses with the left or right hand which, however, were to be executed only if the object's name ended, for example, with an $/ \mathrm{r} /$; the response was to be withheld (nogo condition) when the last letter was an /n/. During performance of this task, the lateralized readiness potential (LRP) was recorded $[8,9,17]$. The LRP is extracted from the electroencephalogram and appears as soon as the primary motor cortex is activated when the decision about the responding hand has been made. The LRP may be present even if the final execution of the overt response is withheld as required in nogo trials [22,24]. If semantic access determines response hand and precedes phonological encoding, as stated by serial models, an LRP should appear even if phonology requires to withhold the response because the go/nogo-related information is available after the decision about the response hand has been made. In fact, Van Turennout et al. [31] did observe LRPs also in nogo trials. Conversely, when the response hand was specified by phonology while semantics determined response execution, there was no LRP in nogo trials. Additional electrophysiological evidence, interpreted in terms of serial models, was presented by Schmitt and coworkers [27] who also recorded the N200 component of the electrical brain response. The N200 emerges in nogo trials and is taken to indicate response inhibition [15,29]. When the nogo decision was linked to semantics, the N200 appeared earlier than when this decision was coupled to name phonology. Both the LRP and N200 findings show that semantic information is available earlier than name phonology, apparently confirming serial models.

Unfortunately, the results of these studies are only informative as to the termination of semantic and phonological retrieval-they are mute as to the relative timing of the beginning of these processes. If semantic retrieval starts simultaneously with phonological encoding but terminates earlier, the same observations in LRPs and N200 as described above are predicted. Therefore, these results are also compatible with parallel/independent retrieval of semantic and name-related knowledge starting simultaneously.

Parallel access to semantic and phonological properties has recently been suggested in models of face identification and naming which-like object naming — can be taken as a special instance of speech production. For the domain of face recognition serial models [5] have been replaced by models which assume that access to a person's name proceeds in parallel to the retrieval of biographical knowledge [7,30]. Both processes are initiated simultaneously, starting from an abstract representation of the person's identity. Interestingly, the function of these abstract representations of persons is similar to the function of abstract concept nodes in models of speech production in that both serve as parallel interfaces for the retrieval of semantic information and object names or names of familiar persons. These models can accommodate two lines of evidence that have traditionally been interpreted in favor of serial models. First, proper name retrieval is typically faster than retrieval of biographical knowledge [16]. Second, whereas spared semantic access in anomia has frequently been reported [12], there is almost no neuropsychological evidence for person naming without semantics. Both kinds of findings can be explained by a scarcity of connections for name nodes to the processing level from which they receive their input [7]. A similar explanation might hold for the lack of evidence for object naming without semantics in neurolinguistic research.

In the experiments reported here, a strong test of serial versus parallel retrieval of semantic and phonological information was performed with a similar task as used by Van Turennout and coworkers. As the most critical feature, our study included a manipulation of the duration of semantic retrieval over two levels of difficulty, which allows to make differential predictions for serial and parallel models. The manipulation of semantic difficulty was accomplished by presenting pictures of animals which were classified either according to their size in real life (small versus large) or according to their diet (herbivore versus carnivore). Furthermore, the animals were also classified according to the initial phoneme of their names (vowel versus consonant). Two experiments were performed with inversed assignments of semantic and phonological attributes to hand and go/nogo decision.

\section{Experiment 1}

In the first experiment, response hand was determined by semantics whereas response execution (go/nogo) depended on name phonology. According to serial models (see Fig. 1) any delays due to increases in semantic difficulty should postpone all subsequent processes to the same degree. Because the LRP emerges when enough semantic information for the initial activation of the correct 
SERIAL MODEL

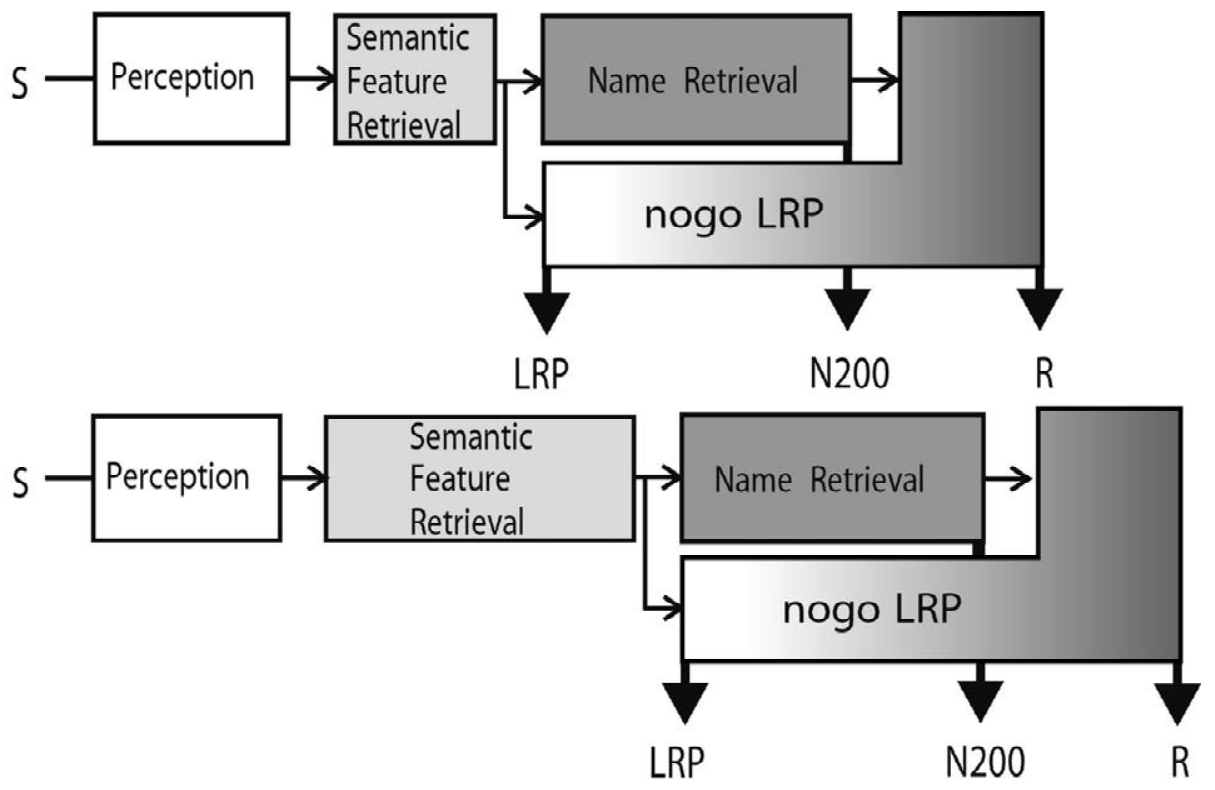

PARALLEL MODEL
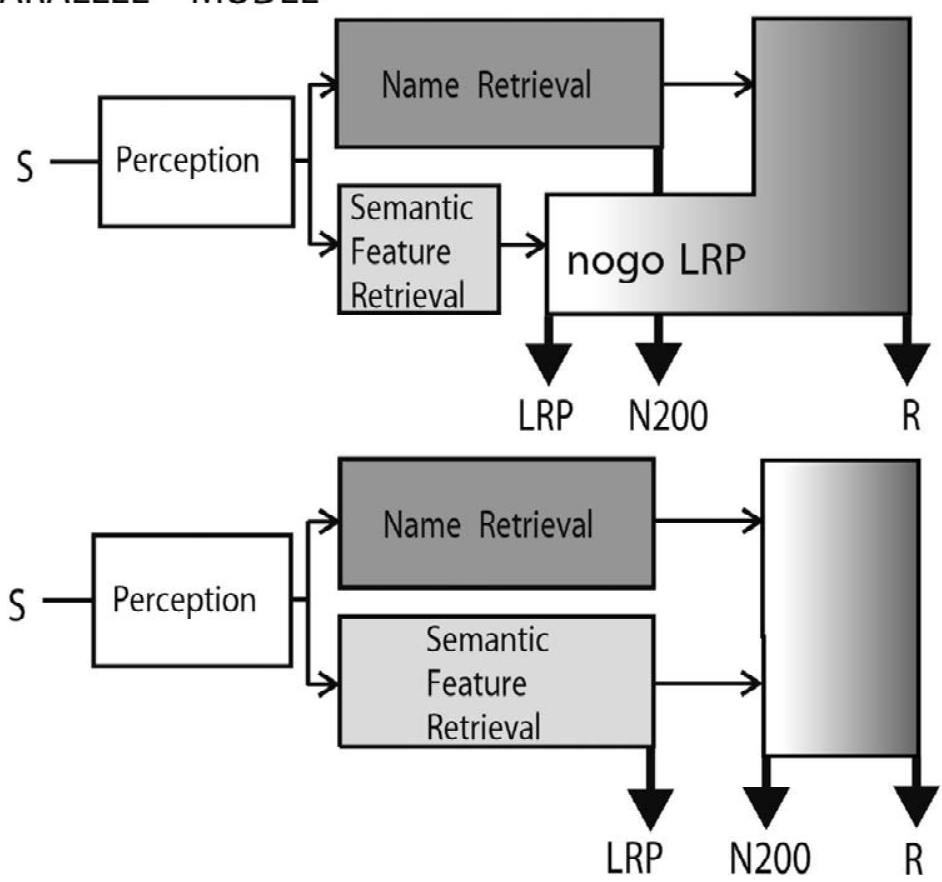

Fig. 1. Schematic predictions of the effects of manipulating semantic difficulty in serial and parallel models of access to semantic knowledge and phonological encoding. In Experiment 1 choice responses with either hand are required as a function of the semantic properties of the stimuli. The onset of hand-specific response activation (hatched box) is indicated by the lateralized readiness potential (LRP). Name phonology determines execution or inhibition of the response (go/nogo). Response inhibition is indicated by the N200 component. In serial models (top panel), the LRP-to-response interval and the presence of an LRP in nogo trials (broken lines in hatched area) are unaffected by varying time demands for semantic access whereas N200 latency varies. Parallel models (bottom panel) predict the LRP-to-response interval and any nogo-LRP to decrease with increasing time demands for semantic processing but there should be no change in N200 latency. 
response hand is extracted, the LRP-to-response interval (for an estimate of this interval the LRP is synchronized to the response ${ }^{1}$ ) should be unaffected by semantic difficulty. In contrast, parallel models predict shorter LRP-to-response intervals for the more difficult semantic condition because, as difficulty increases, the time between the terminations of semantic and phonological access should decrease. In the current tasks the presence of a nogo-LRP depends on the interval between the termination of semantic and phonological encoding. According to serial models this interval should be unaffected by semantic difficulty, whereas in parallel models the interval should decrease as difficulty is raised and likewise the nogo-LRP should decrease. Finally, according to serial models the latency of the N200 elicited in nogo trials, should be longer for difficult than for easy semantic classifications, whereas for parallel models there should be no difference.

In addition to the differential predictions that allow to discriminate between serial and parallel processing, both models predict an effect of semantic difficulty on stimuluslocked LRP onset latency. This effect reflects the longer duration of semantic retrieval for the more difficult diet classification. No predictions were derived for semantic difficulty effects on response times because, again, these effects are in line with both models (cf. Results and discussion).

\subsection{Participants}

Sixteen right-handed native German speakers participated ( 8 women, mean age $=25$ years). All participants reported normal hearing and normal or corrected-to-normal vision. They were either paid for participation or received partial fulfillment of a curriculum requirement.

\subsection{Materials}

The stimulus set consisted of 3 color images each of eight different animal species, namely Orang Utan, Ice Bear (Ger.: Eisbär), Hedgehog (Igel), Camel (Kamel), Ermine (Hermelin) Mouse (Maus), Squirrel (Eichhörnchen) and Leopard. Pictures showed various views of the animals but were scaled to approximately the same size within the frame. Animals could be semantically categorized according to their real size (large versus small), and diet (purely herbivorous versus at least partially carnivorous), and on the initial phoneme of their name (vowel

\footnotetext{
${ }^{1}$ By computing stimulus- and response-locked LRPs experimental effects can be localized on stages before and after response activation. For example, in the current experiment the onset latency of the LRP synchronized to the stimulus is an estimate of the time demand for semantic retrieval. Estimates of the effects of semantic manipulation on the time demands of phonological encoding are provided by the LRP time-locked to the response. Together, both measures provide distinct information about the relative timing of semantic and phonological retrieval.
}

versus consonant). Within dimensions, categories were exclusive and equiprobable, across dimensions they were independent.

\subsection{Pretest}

Ease of categorization was assessed in a separate pretest with 6 different participants and the same basic type of procedure as in the experiments proper. The pictures were presented equiprobably in random order at the center of a color monitor under a visual angle of 2.0 by $2.0^{\circ}$. Each trial started with a fixation cross presented for $0.5 \mathrm{~s}$ in the middle of the screen, followed for $1.3 \mathrm{~s}$ by a picture; the next trial started $1.8 \mathrm{~s}$ later. In three separate counterbalanced blocks of conditions speeded 2-choice responses were performed on each of the three dimensions. Responses were recorded with force-sensitive keys mounted behind each other in the midsagittal plane of the participants. A response was registered as soon as response force exceeded a criterion of $90 \mathrm{cN}$. Mean reaction times for the dimensions size, diet, and initial phoneme were 544, 678, and $681 \mathrm{~ms}$, respectively (error rates: $M=5.5,10.9$, and $10.2 \%$, respectively). The large difference in temporal processing demands between the semantic classification tasks (size versus diet; $t(5)=7.9 ; P<0.001$ ) is a necessary prerequisite for the experiment proper.

\subsection{Procedure}

In the beginning we made sure that each participant knew the relevant semantic and phonological properties for each stimulus picture. In Experiment 1, the semantic dimensions determined the responding hand whereas the phonological dimension determined whether the response was to be executed or not (go versus nogo). The order of semantic conditions (size, diet) and assignment of semantic attribute to response finger was counterbalanced. The go/ nogo decision was coupled to phonology (vowel or consonant as initial phoneme) in block wise alternation, also counterbalanced across subjects.

The experiment contained a total of 1632 trials, subdivided into 8 blocks of equal length. Stimulus presentation proceeded as in the pretest. To encourage response preparation, stimuli associated with response execution (go stimuli) were presented four times as often as nogo stimuli [21]. Because the go/nogo assignment of the alternative categories alternated between blocks, all stimuli appeared equiprobably with respect to the whole experiment. In order to encourage phonological encoding and to prevent the use of automatic stimulus-response assignments $20 \%$ of the pictures had to be named verbally which was indicated by a tone, following the picture. Eye movements or blinks were to be avoided and feedback was given for false alarms on nogo trials, wrong key-presses and omissions on go trials. 


\subsection{Electrophysiological recordings and data analysis}

The EEG was recorded with a right-mastoid reference at the scalp positions $\mathrm{Fz}, \mathrm{Cz}$ and $\mathrm{Pz}$, as defined by the international 10-20 system [14] and from two electrode sites $4 \mathrm{~cm}$ to the left and right of $\mathrm{Cz}$ (C'3 and $\left.\mathrm{C}^{\prime} 4\right)$. In addition, we recorded the horizontal and vertical electrooculogram, the electromyogram (EMG) from standard electrode placements for finger flexor muscles at both forearms [20] and response force. Electrode impedance was kept below $5 \mathrm{k} \Omega$ for cephalic sites and below $10 \mathrm{k} \Omega$ for EMG electrodes.

The signals were sampled at a rate of $250 \mathrm{~s}^{-1}$ after low-pass filtering at $40 \mathrm{~Hz}$. Offline, the continuous record was intersected into epochs of $1.5 \mathrm{~s}$ centered around stimuli and responses. Stimulus- and response-synchronized epochs began 200 and $900 \mathrm{~ms}$ prior to stimulus and response onset, respectively. For the analyses only trials were used with correct responses within a time window of $1300 \mathrm{~ms}$ after stimulus onset and without ocular artifacts (blink threshold $30 \mu \mathrm{V}$ ) or EMG artifacts. Trials with amplifier blocking or shifts greater $120 \mu \mathrm{V}$ within any channel were discarded.

Electrophysiological signals were averaged separately for the easy and difficult semantic conditions and for go and nogo trials. LRP was calculated from the C'3 and C' 4 electrodes as difference waves between the site contralateral and ipsilateral to the response hand. These difference waves were averaged across left- and right-hand responses. An analogous computation was also performed for hEOG, EMG, and response force in order to facilitate comparisons and to assess possible EOG artifacts on the LRP.

Baseline corrections of the ERPs were made by subtracting the average voltage during a $200 \mathrm{~ms}$ interval, immediately before the stimulus for stimulus-synchronized waveforms and preceding response onset by $700 \mathrm{~ms}$ for response-synchronized waveforms. The onset latencies of stimulus- and response-synchronized LRPs were measured with an absolute criterion of $0.5 \mu \mathrm{V}$ below baseline. Latency differences between conditions were statistically tested with a jackknife-based technique [23]. The presence of an LRP on nogo trials was assessed by two-tailed $t$-tests against zero for the mean LRP amplitude at consecutive 20 ms time intervals, starting $100 \mathrm{~ms}$ after stimulus onset. An LRP was considered to be present if at least three successive $20 \mathrm{~ms}$ intervals differed significantly $(P<0.05)$ from zero.

The N200 component was isolated at the Fz electrode by subtracting ERPs from the go and nogo conditions for each semantic condition. The vEOG was computed analogously. N200 latency was measured as the time point of the minimum in the waveform between 100 and $600 \mathrm{~ms}$.

\section{Results and discussion}

Whereas there was no difference in error rates $(M=5.1$
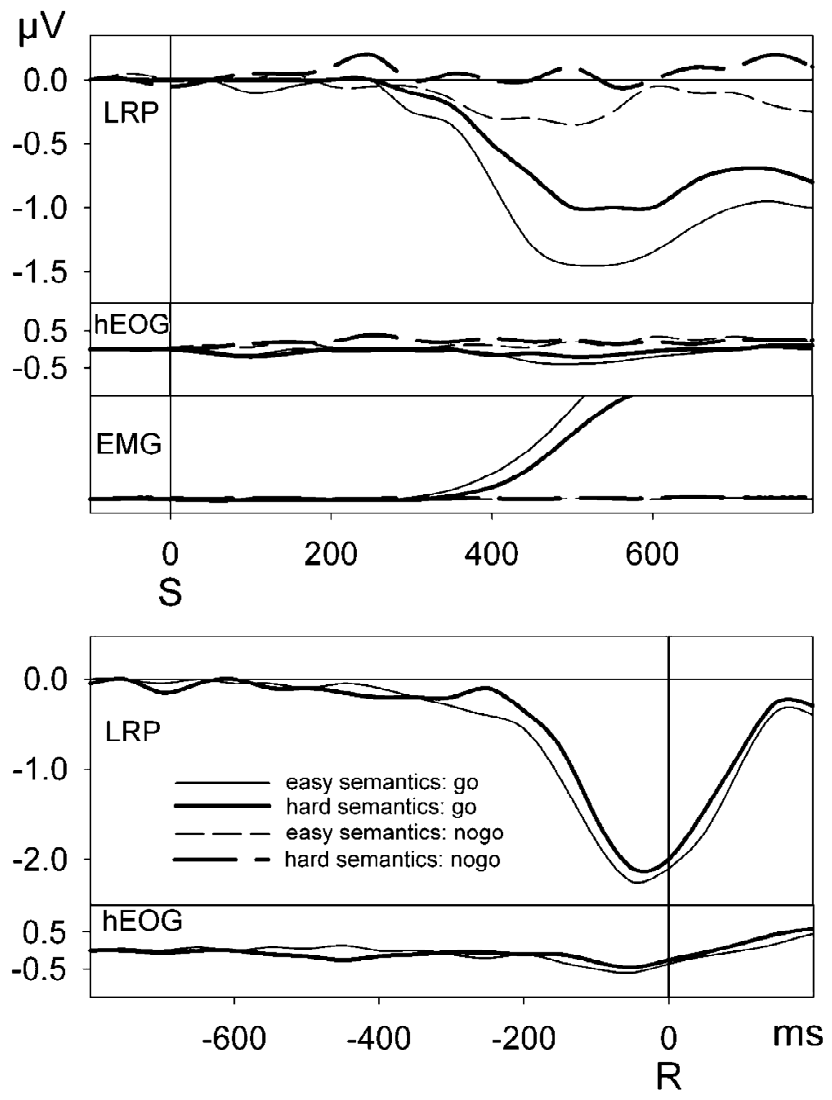

Fig. 2. Stimulus- and response-synchronized (top versus bottom panel) LRP waveshapes, hEOG, and EMG activity computed analogously, in Experiment 1 as a function of semantic difficulty. $\mathrm{S}$ and $\mathrm{R}$ denote onsets of stimuli and responses, respectively.

versus $6.6 \%$ ), reaction times (RT) in go-trials were shorter in the size classification than in the diet classification $(M=651$ versus $692 \mathrm{~ms}), t(15)=-3.9 ; P<0.001$. As predicted by both serial and parallel models the stimulussynchronized LRP (Fig. 2, top) started earlier in the easy than in the difficult semantic condition $(M=355$ versus 390 $\mathrm{ms}$ ), $t(15)=1.8 ; P<0.05$ (one-tailed) ${ }^{2}$. Whereas there was a sizeable nogo-LRP in the easy semantic condition, lasting for $80 \mathrm{~ms}, t \mathrm{~s}>-2$, no such activity was present in the difficult condition. Relative to the response (Fig. 2, bottom), LRP started earlier in the easy than in the difficult semantic condition $(M=-245$ versus $-180 \mathrm{~ms}), t(15)=$ $2.9 ; P<0.01$. The latency of the N200 component (Fig. 3) did not differ significantly between the easy and difficult semantic condition $(M=415$ versus $385 \mathrm{~ms}), t<-0.7$.

\footnotetext{
${ }^{2}$ The amplitude of the stimulus-locked LRP is larger in the easy than in the hard semantic condition. This effect can most likely be attributed to a greater latency variability of the individual LRP waveforms for the difficult semantic condition. Such latency variability tends to flatten the slopes of average ERP waveforms and to diminish their amplitudes. This explanation is supported by the observation that slope and amplitude differences are much smaller in our response-locked averages (cf. Fig. 2, bottom) where latency variability is usually minimal.
} 


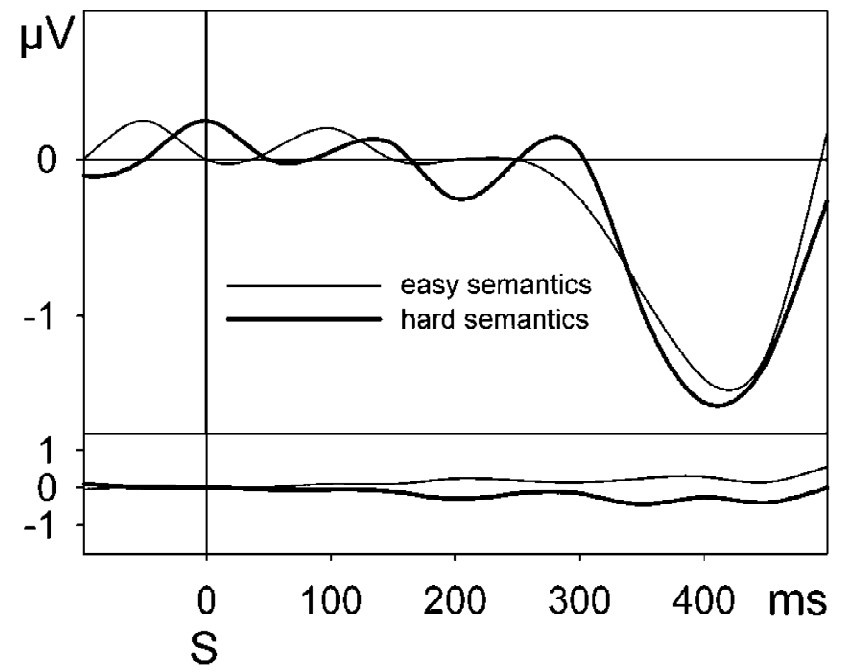

Fig. 3. Difference waves derived by subtracting ERPs and vEOG in goand nogo-conditions as a function of semantic difficulty in Experiment 1. The negative deflection around $400 \mathrm{~ms}$ is the N200 component, considered to indicate response inhibition.

Three differential predictions from serial as compared to parallel models of access to semantic knowledge and phonological encoding were made for the effects of semantic difficulty on electrophysiological variables. In all three cases the results obtained were in line with parallel and at variance with serial models. Increasing semantic difficulty: (1) decreased the response-locked LRP onset latency, (2) obliterated the nogo-LRP activity but (3) did not affect N200 latency. These results cannot be attributed to an inefficient manipulation of semantic difficulty because, as predicted by both types of models, there was a clear effect on the stimulus-to-LRP interval.

Somewhat unexpectedly, the nogo-LRP in the easy semantic condition developed later than the go-LRP (cf. Fig. 2). We assume that this is due to a relatively high proportion of go- as compared to nogo-stimuli in the present experiment. This is a common procedure in the LRP literature [21] but differs from those used in other speech production experiments with LRPs [27,31], making direct comparisons between studies more difficult. However, with respect to our differential predictions for the occurrence of a nogo-LRP in the two semantic conditions the higher proportion of go stimuli is not critical because it holds for both semantic conditions and can not explain why the nogo-LRP vanished in the more difficult condition.

The interpretation of RTs is less clear. Serial models predict an effect of semantic difficulty whereas parallel models, as depicted schematically in Fig. 1, appear to indicate a complete absorption of the semantic difficulty effect in RTs. Therefore, the reaction time effects of semantic difficulty appear to meet the prediction of serial models only. However, the observed effect is also compatible with a parallel architecture. One need only assume that parallel to the on-going phonological retrieval, participants already prepare the response hand determined by the semantic information. In this case, the easy semantic condition will gain an advantage over the difficult one because of the possibility to prepare the response in advance. This explanation is in line with the exclusive presence of a nogo-LRP in the easy semantic condition. That there was some absorption of the difficulty effect in RTs in the two-choice go/nogo condition of Experiment 1 is indicated by the fact that the effect had been much more pronounced in the 2 -choice pretest $\left(M_{\text {diff }}=41\right.$ versus 134 $\mathrm{ms})$. According to serial models the effect of semantic difficulty should have been of a comparable magnitude in both tests.

Another conceivable reason for an RT effect within a parallel architecture is the possible overlap of the temporal distributions for the termination of semantic retrieval and phonological encoding. Because information from both processes has to be available before the correct response can be carried out, mean RT is a function of the terminations of both processes. More specifically, the cumulated probability of a response at a given latency is a function of the product of the cumulated probabilities for the termination of semantic and phonological retrieval at this latency. If the distributions of both processes are non-overlapping, as may be expected for a combination of easy semantics with name classification, the RT distribution is only a function of the distribution of the slower process (name classification) because in this case, semantic retrieval has always finished before phonological encoding has. If, in contrast, the two distributions overlap, as may be expected for a combination of difficult semantics with name classification, their product, the overall mean RT distribution will be slowed as compared to the single distributions of the two processes in isolation.

It might also be argued that the present results are due to a task artifact. Possibly, the task might have compelled the participants to always make the hand decision prior to the go/nogo decision, which imposes a certain order of processing. However, even if this had been the case, it would not explain the differential effects of semantic difficulty on response-locked LRP onset latency and on the nogo-LRP.

Alternatively, can the results be explained by an influence of task difficulty or by the fact that, in contrast to pure picture naming, additional decision processes are involved in the present task? Which classification is made first might be determined by difficulty, regardless of the decision required. In our view, a strategy of retrieving the more accessible information first is much more feasible in a parallel system. To accommodate such a strategy in a serial model would require the awkward assumption that participants wait till both kinds of information are available and then use the more accessible information first. An important control against the task artifact argument will be provided by Experiment 2 where also a further differential prediction was tested. 


\section{Experiment 2}

Here, the assignment of semantic and phonological properties to hand and go/nogo decision was reversed; that is, response hand was determined by name phonology whereas response execution (go/nogo) depended on the semantic dimensions. Because according to serial models response hand in Experiment 2 is activated after phonological classification, the LRP in the easy semantic condition should develop earlier than in the difficult semantic condition (Fig. 4). In contrast, if retrieval of phonology proceeds independently of semantic retrieval as proposed by parallel models, semantic difficulty should not affect LRP onset latency. It should, however be reflected in N200 peak latency because the nogo decision is made earlier in the easy semantic condition.
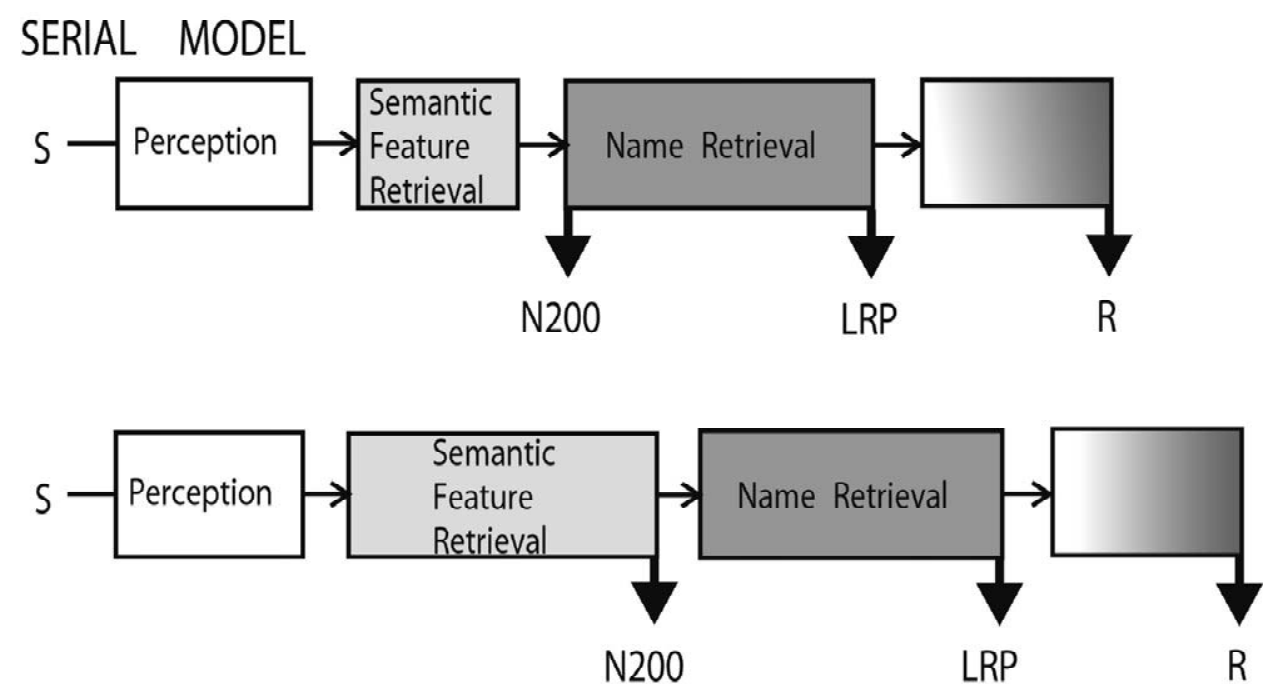

PARALLEL MODEL
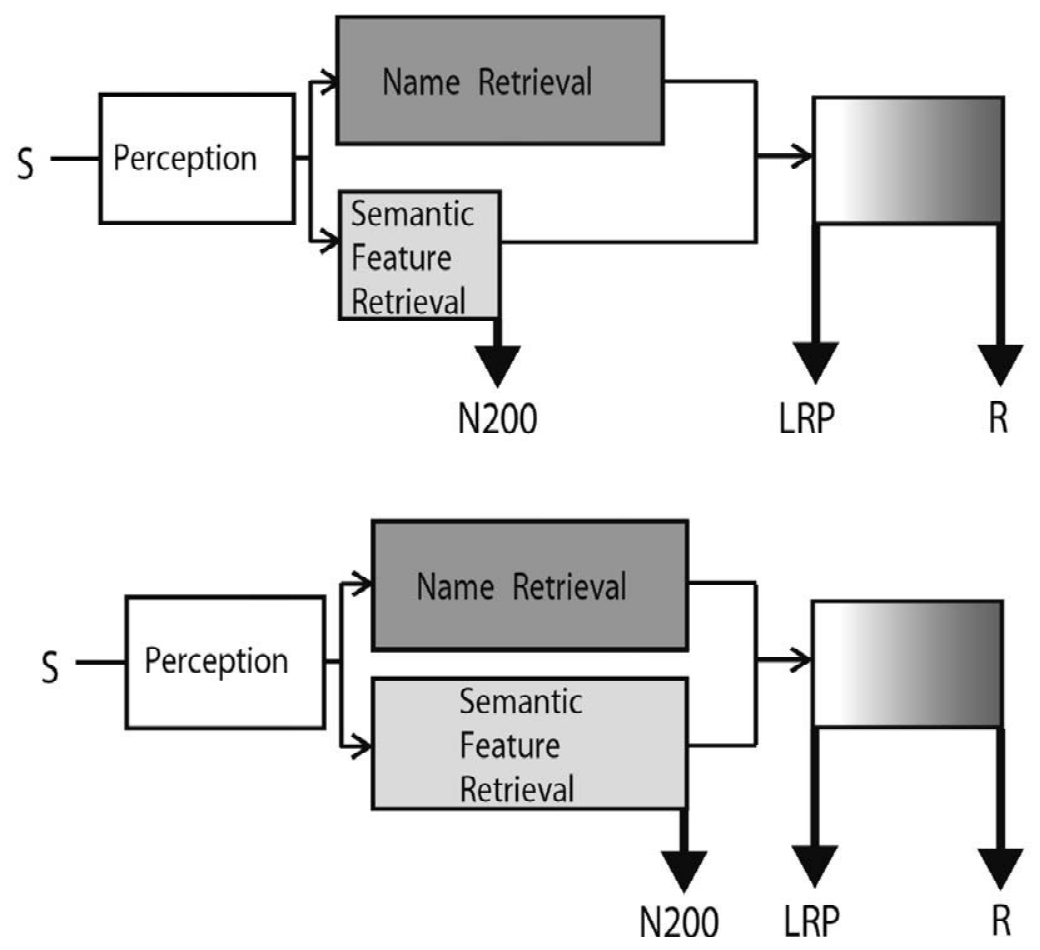

Fig. 4. Schematic predictions for reversed task assignment (Experiment 2); LRP-inducing choice responses are coupled to name phonology whereas execution or inhibition (N200) is determined by semantic properties. In serial models (top panel) the stimulus-to-LRP interval and N200 peak latency should increase with semantic difficulty. According to parallel models (bottom panel) there should be no effect of semantic difficulty on the stimulus-to-LRP interval, whereas N200 latency should increase. 


\subsection{Participants}

Sixteen right-handed native German speakers took part ( 12 women, mean age $=21$ years) in exchange for payment. All participants reported normal hearing and normal or corrected-to-normal vision.

\subsection{Materials}

The same stimulus set as in Experiment 1 was used.

\subsection{Procedure}

The procedure was identical to Experiment 1 except for task instructions. The phonological classification determined response hand and the semantic classification determined the go/nogo decision.

\subsection{Electrophysiological recordings and data analysis}

The electrophysiological recording and data analysis was identical to that in Experiment 1.

\section{Results and discussion}

Again, there was no difference in error rates $(M=3.3$ versus $4 \%$ ), whereas reaction times in the easy semantic condition were shorter than in the difficult condition $(M=$ 646 versus $675 \mathrm{~ms}), t(15)=-5.1 ; P<0.001$. The LRP onset latencies for the easy and difficult semantic condition $(M=396$ versus $388 \mathrm{~ms}$; cf. Fig. 5) did not differ as a function of semantic difficulty, $t(15)=-0.4$. The LRP waveforms on nogo trials did not approach statistical significance $t \mathrm{~s}<-1.7$. As predicted by both serial and parallel models, N200 latency (Fig. 6) was shorter in the

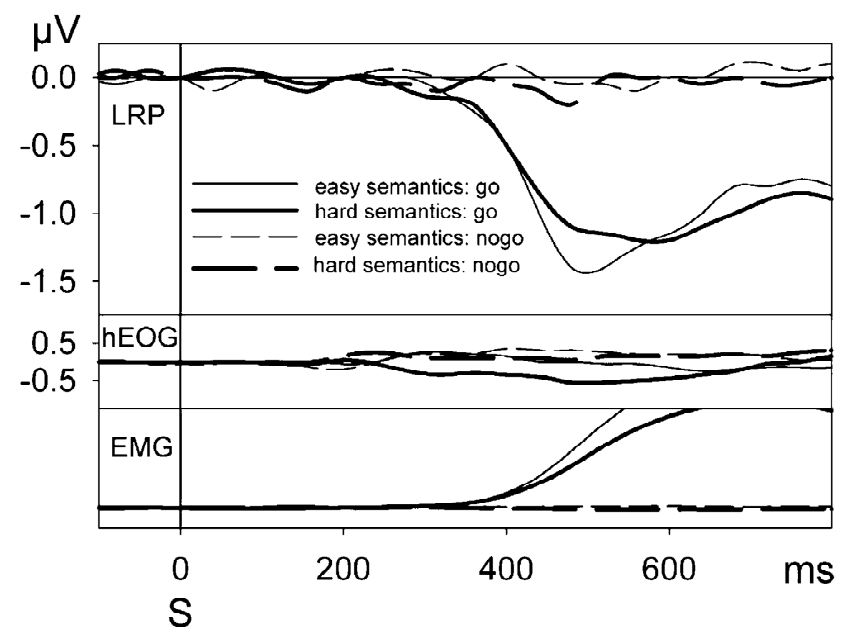

Fig. 5. Stimulus-synchronized LRP waveshapes, hEOG, and EMG from Experiment 2 as a function of semantic difficulty. $\mathrm{S}$ denotes stimulus onset.

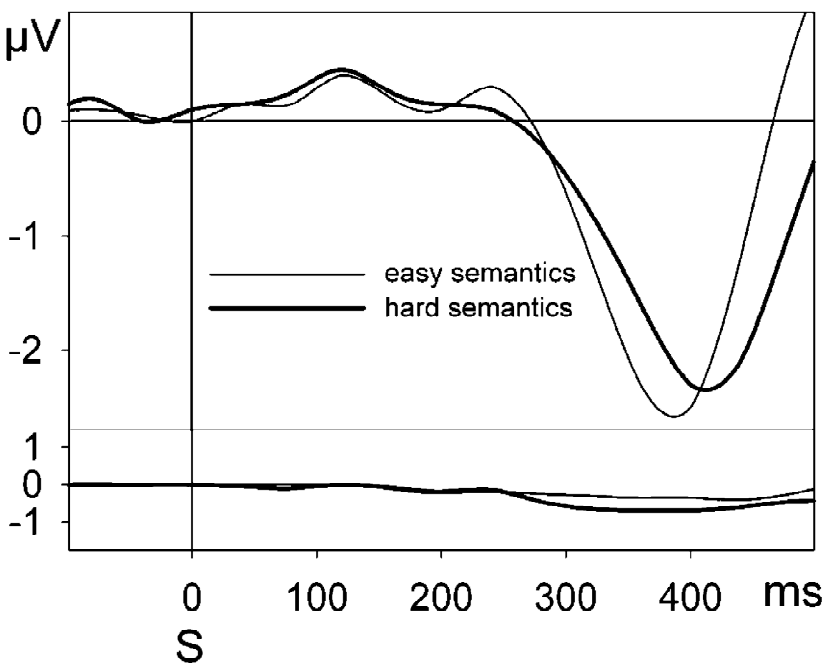

Fig. 6. Difference waves derived by subtracting ERPs and vEOG in go and nogo conditions as a function of semantic difficulty in Experiment 2. The negative deflection around $400 \mathrm{~ms}$ is the N200 component, considered to indicate response inhibition.

easy as compared to the difficult semantic condition $(M=$ 380 versus $408 \mathrm{~ms}), t(15)=-3.7 ; P<0.002$.

For this experiment there was only one differential prediction for the effect of semantic difficulty; whereas serial models predict an increasing stimulus-to-LRP interval for the easy as compared to the difficult semantic condition, parallel models do not predict any changes for this interval. As before, the result was in favor of parallel access.

Reaction times and N200 latency in Experiment 2 again confirm the efficiency of the difficulty manipulation. This experiment also demonstrates that the 2-choice go/nogo task employed here does not necessarily impose an order of processing, for example, requiring hand decisions to be made prior to go/nogo decisions. If this were the case one would have expected nogo-LRPs also in this experiment. A further argument against a task-imposed order of processing are the differential effects of the experiments on the stimulus-to-LRP intervals and N200 peak latencies. We can, thus, conclude that the temporal requirements of the duration of semantic retrieval were manipulated successfully.

\section{General discussion}

Using electrophysiological measures we addressed the question whether retrieval of semantic and phonological knowledge in an object identification and naming task proceeds in series or in parallel. The presence of a nogoLRP when response hand was determined by (easy) semantics (Experiment 1) but not when it was determined by name phonology (Experiment 2 ) replicates findings by Van Turennout et al. [31] and Schmitt and coworkers [27] 
which had been interpreted in favor of serial models. However, manipulation of the semantic difficulty in the present experiments provided evidence for parallel retrieval of semantic knowledge and name phonology. For all four differential predictions (three in Experiment 1 and one in Experiment 2) empirical results consistently supported those derived from parallel models, indicating that retrieval of phonology is not contingent on prior retrieval of the semantic features required to perform the present task; instead, phonological encoding appears to start at the same time as semantic retrieval. The nogo-LRP results of Van Turennout et al. and Schmitt et al. can be easily reconciled with the present findings by assuming that under the conditions of their studies, similarly to the easy condition in the present experiments, semantic retrieval terminates before phonological encoding does, allowing for the appearance of a nogo-LRP when hand decisions are determined by semantics.

As outlined in the Introduction, the nogo-LRP clearly indicates that some semantic features, like animacy or size, are available for response activation while the executionrelated (phonological) information is not yet extracted. Serial models predict that a nogo-LRP under these conditions should be independent of the duration of semantic retrieval. In contrast, in a parallel arrangement a nogo-LRP is predicted only when semantic properties are determined earlier than is phonological information. Therefore, we introduced the difficulty manipulation of semantic retrieval which abolished the nogo-LRP activity as predicted by parallel models when the slack between the ends of semantic and phonological processes is diminished. Even more important than the differential appearance of a nogoLRP is the variation of the response-locked LRP onset latency as a function of semantic difficulty. Only parallel models predict the counterintuitive effect of a shortened interval between LRP onset and the response when semantic processing requires more time. Note that present RTs were not taken as evidence for one of the two models because they are in line with both serial as well as parallel processing models (cf. Results and discussion Experiment $1)$.

A possible concern is related to the LRP as indicating only the termination of a preceding process. It is conceivable that the semantic (Experiment 1) and phonological (Experiment 2) information required for the hand decision is transmitted continuously to the LRP-generating motor stages whereas, in line with serial discrete models of speech production, name retrieval may depend on the completion of semantic retrieval. However, in this case the chances for the presence of a nogo-LRP in the difficult semantic condition in Experiment 1 would even increase because there would be as much or even more time to transmit information to motor stages than in the easy condition. This is clearly at variance with the absence of a nogo-LRP in the difficult semantic condition of Experiment 1 .
One might ask whether the restricted number of different stimuli used in the present experiments might cause direct mappings between stimuli and responses and, as a consequence, supersede retrieval of the concrete semantic and phonological information. However, such a direct stimulus-response $(\mathrm{S}-\mathrm{R})$ mapping should obliterate any effect of semantic difficulty. This was obviously not the case because semantic difficulty did affect the LRP onset latency in Experiment 1 and also N200 peak latency in Experiment 2. Therefore, our precautions against direct $\mathrm{S}-\mathrm{R}$ mappings, namely the presentation of three different photographs for each animal and interspersed naming trials, appear to have been successful. One might also assume that with extensive practice participants learn to directly classify the stimuli as having names with or without initial vowels without actually retrieving the name, whereas semantics might still be retrieved. In this case one would expect LRP activity in nogo trials of Experiment 2 at least in the difficult semantic condition because the automatic name classification should be faster than retrieval of semantic features. Conversely, in Experiment 1 no nogo-LRP should show up because here the nogo-decision should have been based on a fast automatic classification, finished before semantic information becomes available. As reported, both predictions from such a hybrid conception were not borne out.

Might the employment of initial phonemes as classification criterion have biased the present results? From tip-ofthe-tongue research it is known that initial phonemes have a special role in lexical retrieval [4,6]. Therefore, one might suggest that parallel retrieval with semantics is only possible with initial but not with later phonemes which would not be expected in a serial discrete model. Although there appears to be no direct evidence for this possibility, it can be addressed by applying the present design. Taken together, some aspects of the present results, like the nogo-LRP in the easy condition and the LRP onset latency differences of Experiment 1 and the RT effects in Experiments 1 and 2, comply with both parallel as well as serial models. However, the overall pattern of those results that are relevant for the differential predictions discriminating between these models is consistently in line with parallel models only.

Experiments 1 and 2, together with the findings of Van Turennout and colleagues [31] and Schmitt and colleagues [27] reveal two lines of evidence. Semantic features like animacy or size are available earlier than phonological information about the object name. However, the asynchrony in availability does not necessarily imply seriality of these processes. Manipulating the duration of semantic feature retrieval did not affect the beginning of word form encoding, as predicted by serial models. Therefore, a fully serial model in which all stored semantic features have to be retrieved in order to name an object has to be ruled out. There are at least two alternatives to accounts for the present results. 
First, one might suggest a hybrid architecture in which certain basic or critical semantic features mediate word form encoding, whereas other non-critical semantic features can be retrieved in parallel to the word forms. Applied to the present experiments at least the difficult semantic feature (diet) or even both features (size plus diet) might be non-critical in this sense, yielding the present results that indicate parallel retrieval. Unfortunately, the empirical and theoretical basis for a specification of semantic retrieval as required for object naming seems to be weak and one would be hard-pressed to make any suggestions as to define which semantic features might be critical or non-critical. In speech production models, it is usually not specified which semantic features are necessary for object naming. In fact, the production system might be flexible in a way that, given a specific context (e.g., intended message), any feature might be critical or noncritical. Thus there is a clear need for more research on which semantic properties do and which do not play a critical role for object naming; or whether semantic mediation can be modulated by providing context information. The present design appears to be a useful tool for this purpose and provides first evidence that semantic processing might indeed be lean. Recently, Abdel Rahman, Van Turennout and Levelt [2] replicated and extended the present findings with a more commonly accepted basic semantic feature (animacy). The retrieval speed of this feature was selectively manipulated with a semantic task mixing procedure. In line with the present findings the results suggest that a basic semantic feature like animacy, although retrieved prior to name phonology, is not essential for the initiation of phonological encoding

The second alternative to accommodate the present findings is a purely parallel account suggesting that semantic feature retrieval and phonological encoding are initiated independently. As described in the introduction, theories of face recognition and naming [1,6,29] suggest such parallel activation for semantic (biographical) knowledge and names of persons. Common starting point of these activations are abstract person identity nodes which are not stores of concrete knowledge but merely nodes that direct the flow of activation. In analogy, one might suggest abstract representations of objects which are accessed during object identification and naming. After the activation of these 'object identity nodes' there can be parallel retrieval of various kinds of concrete semantic knowledge and name phonology. This suggestion may be extended to speech production in general if the abstract representations of objects are seen as instances of abstract lexical concept nodes. As described in the introduction, the idea of an abstract conceptual representation in models of word production is by no means a new one. It is expressed in non-decompositional views of conceptually driven lemma retrieval $[19,25,26]$. These theories claim that lexical concepts are not represented by sets of semantic features which, in combination, constitute the meaning of a word but as undivided wholes. Concrete semantic features that represent word meaning are retrieved via labeled links to other concept nodes. For example, the concept ice bear is an abstract representation which is used, on the one hand, to retrieve semantic properties like ANIMATE or CARNIVOROUS and, on the other hand, to activate the appropriate lexical candidate for a naming response. Within a framework of non-decomposed concept representations, our data suggest that retrieval of concrete semantic features from an abstract concept node can proceed in parallel to lexical access proper. In this arrangement, the time course of semantic feature retrieval might differ between features and it might be faster than name retrieval in most cases (as indicated by the nogo-LRP elicited by semantic classification) without requiring that picture naming is necessarily mediated by prior retrieval of concrete semantic features.

In general, the current experiments demonstrate that the LRP methodology in combination with the appropriate experimental design is well-suited to distinguish between serial and parallel processing, a question which arises not only in the area of language production but also in many other areas such as face recognition [1]. For the domain of speech production, the present study can be taken as a starting point for further investigations on the interplay of different types of mental operations during speaking with electrophysiological measures. For example, it could be used to distinguish between serial and parallel views of the organization of syntactic and phonological encoding.

\section{Acknowledgements}

This research was supported by $\mathrm{Ph} . \mathrm{D}$. fellowship (Graduate School 423) from the German Research Council to Rasha Abdel Rahman. We appreciate encouraging and helpful discussions with Herbert Schriefers and thank Matti Laine and an anonymous reviewer for constructive suggestions on an earlier version of the paper.

\section{References}

[1] R. Abdel Rahman, W. Sommer, S.R. Schweinberger, Brain potential evidence for the time course of access to biographical facts and names of familiar persons, J. Exp. Psychol. 28 (2002) 366-373.

[2] R. Abdel Rahman, M. Van Turennout, W.J.M. Levelt, Phonological encoding is not contingent on semantic feature retrieval: an electrophysiological study on object naming, J. Exp. Psychol., in revision.

[3] M. Bierwisch, R. Schreuder, From lexical concepts to lexical items, Cognition 42 (1992) 23-60.

[4] A. Brown, A review of the tip-of-the-tongue experience, Psychol. Bull. 109 (1991) 204-223.

[5] V. Bruce, A. Young, Understanding face recognition, Br. J. Psychol. 77 (1986) 305-327.

[6] D.M. Burke, D.G. MacKay, J.S. Worthley, E. Wade, On the tip of the tongue: what causes word finding failures in young and older adults?, J. Mem. Lang. 30 (1991) 542-579. 
[7] A.M. Burton, V. Bruce, R.A. Johnston, Understanding face recognition with an interactive activation and competition model, Br. J. Psychol 81 (1990) 361-380.

[8] M.G.H. Coles, Modern mind-brain reading: psychophysiology, physiology and cognition, Psychophysiology 26 (1989) 251-269.

[9] R. De Jong, M. Wierda, G. Mulder, L.J.M. Mulder, Use of partial stimulus information in response processing, J. Exp. Psychol. 14 (1988) 682-692.

[10] G.S. Dell, A spreading-activation model of retrieval in sentence production, Psychol. Rev. 93 (1986) 283-321.

[11] G.S. Dell, P.G. O'Seaghdha, Stages of lexical access in language production, Cognition 42 (1992) 287-314.

[12] J.R. Hodges, K. Patterson, N. Graham, K. Dawson, Naming and knowing in dementia of Alzheimer's type, Brain Lang. 54 (1996) $302-325$.

[13] P. Indefrey, W.J.M. Levelt, The neural correlates of language production, in: M.S. Gazzaniga (Ed.), The Cognitive Neurosciences, MIT Press, Cambridge, MA, 2000, pp. 845-865.

[14] H.H. Jasper, The ten-twenty electrode system of the international federation, Electroenc. Clin. Neurophys. 10 (1958) 371-375.

[15] E. Jodo, Y. Kayama, Relation of a negative ERP component to response inhibition in a Go/No-go task, Electroenc. Clin. Neurophys. 82 (1992) $477-482$.

[16] R.A. Johnston, V. Bruce, Lost properties? Retrieval differences between name codes and semantic codes for familiar people, Psychol. Res. 52 (1990) 62-67.

[17] M. Kutas, E. Donchin, Preparation to respond as manifested by movement-related brain potentials, Brain Res. 202 (1980) 95-115.

[18] W.J.M. Levelt, Speaking: From Intention to Articulation, MIT Press, Cambridge, MA, USA, 1989.

[19] W.J.M. Levelt, A. Roelofs, A.S. Meyer, A theory of lexical access in speech production, Beh. Brain Sci. 22 (1999) 1-75.

[20] O.C.J. Lippold, Electromyography, in: P.H. Venables, I. Martin (Eds.), A Manual of Psychophysiological Methods, Elsevier, New York, 1967, pp. 245-297.
[21] K.A. Low, J. Miller, The usefulness of partial information: effects of go probability in the choice/gogo task, Psychophysiology 36 (1999) 288-297.

[22] J. Miller, S.A. Hackley, Electrophysiological evidence for temporal overlap among contingent mental processes, J. Exp. Psychol. 121 (1992) 195-209.

[23] J. Miller, T. Patterson, R. Ulrich, Jackknife-based method for measuring LRP onset latency differences, Psychophysiology 35 (1998) 99-115.

[24] A. Osman, T.R. Bashore, M.G.H. Coles, E. Donchin, D.E. Meyer, On the transmission of partial information: inferences from movement-related brain potentials, J. Exp. Psychol. 18 (1992) 217232.

[25] A. Roelofs, A spreading-activation theory of lemma retrieval in speaking, Cognition 42 (1992) 107-142.

[26] A. Roelofs, A case of nondecomposition in conceptually driven word retrieval, J. Psychol. Res. 26 (1997) 33-67.

[27] B.M. Schmitt, T.F. Münte, M. Kutas, Electrophysiological estimates of the time course of semantic and phonological encoding during implicit picture naming, Psychophysiology 37 (2000) 473-484.

[28] B.M. Schmitt, K. Schilz, W. Zaake, M. Kutas, T.F. Münte, An electrophysiological analysis of the time course of conceptual and syntactic encoding during tacit picture naming, J. Cogn. Neurosci. 13 (2001) 510-522.

[29] R. Simson, H.G. Vaughan, W. Ritter, The scalp topography of potentials in auditory and visual Go/NoGo tasks, Electroenc. Clin. Neurophys. 43 (1977) 864-875.

[30] T. Valentine, T. Brennen, S. Brédart, The Cognitive Psychology of Proper Names, Routledge, London, 1996.

[31] M. Van Turennout, P. Hagoort, C.M. Brown, Electrophysiological evidence on the time course of semantic and phonological processes in speech production, J. Exp. Psychol. 23 (1997) 787-806.

[32] M. Van Turennout, P. Hagoort, C.M. Brown, Brain activity during speaking: from syntax to phonology in 40 milliseconds, Science 280 (1998) 572-574. 